\title{
Ultrasonographic Application in the Diagnosis and Prognosis of Pericarditis in Cows
}

\author{
Sheikh Imran, S. P. Tyagi, Amit Kumar, Adarsh Kumar, and Shivali Sharma \\ Department of Veterinary Surgery and Radiology DGCN, College of Veterinary and Animal Sciences, CSK HP Agricultural University, \\ Palampur, Himachal Pradesh, 176 062, India \\ Correspondence should be addressed to Sheikh Imran, sheikhimran_08@rediffmail.com
}

Received 20 October 2010; Accepted 5 February 2011

Academic Editor: Sumanta Nandi

Copyright ( $) 2011$ Sheikh Imran et al. This is an open access article distributed under the Creative Commons Attribution License, which permits unrestricted use, distribution, and reproduction in any medium, provided the original work is properly cited.

\begin{abstract}
The present study was conducted on 5 cows diagnosed with late-stage pericarditis based on a combination of clinical, radiographic, ultrasonographic, and postmortem examinations. On clinical examination, all the cows were found to be depressed, dyspnoeic, and moderately to severely tachycardic. The main radiographic findings were poor thoracic details and indistinguishable cardiophrenic silhouette. Ultrasonographically, the pericardial sac contained varying quantities of hypoechogenic exudate and echogenic fibrin shreds, which had caused physical compression of the cardiac chambers. The clinical and radiographic findings did not allow a definitive diagnosis of pericarditis, because all of the characteristic signs were not present in every case. Thoracic and abdominal ultrasonography played an important role in the early diagnosis and prognosis of the disease.
\end{abstract}

\section{Introduction}

Pericarditis includes inflammation of both the parietal and visceral surfaces of the pericardial cavity [1]. Bovine pericarditis is most commonly caused by traumatic foreign bodies infiltrating across the diaphragm, originating in the reticulum. The diagnosis of pericarditis in cattle is generally based on signs, clinical examination, pericardiocentesis, and thoracic radiography. The most consistent findings of pericarditis are tachycardia, muffled heart sounds, and jugular venous distention/pulsation [2], although clinical presentation varies with the volume of pericardial effusion. The aforementioned signs may also be observed in many other diseases such as tachycardia in dehydration, hypovolemia, anemia, hypoxemia, fever, acid-base and metabolic disturbance, pain, excitement, stress, sepsis or toxemia [3], muffled heart sounds in pulmonary emphysema, pneumothorax or lymphosarcoma with pericardial involvement, and jugular venous distention/pulsation in diaphragmatic hernia, cardiomyopathy, tricuspid insufficiency, jugular venous phlebitis/thrombosis, cardiac abscess/tumour, or cor pulmonale caused by chronic pneumonia $[2,4]$.

Therefore, a straightforward diagnosis of the pericarditis may be precluded due to the ambiguous nature of its clinical signs. Pericardiocentesis plays a major role in differential diagnosis; however, it is not without risk, as potential complications may develop such as pneumothorax, fatal arrhythmia, cardiac puncture leading to haemorrhage or death, and leakage of pericardial material into the thorax, resulting in pleuritis [5]. Poor details of the thoracic radiographs due to radiodense exudate in chronic stage of pericarditis increase the chances of misdiagnosis with the other conditions such as pleural effusion.

Being a major challenge in bovine cardiology, pericarditis has been discussed in great detail [5-9]; however, very few ultrasonographic studies on pericarditis have been published until now $[10,11]$. In the present study, ultrasonographic examinations of the thorax and abdomen were carried out in cows with pericarditis, in order to determine the extent of the lesions and to assess better the prognosis. The results were compared with the findings 


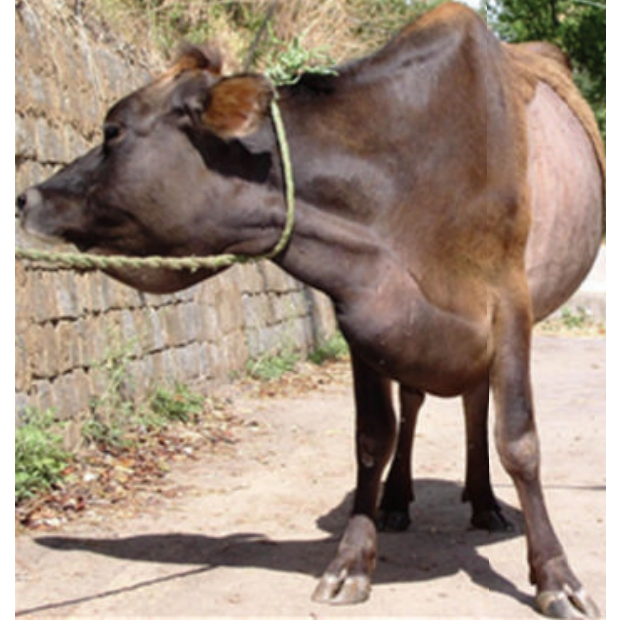

Figure 1: Ventral edema and jugular venous distention in a Jersey cross-bred cow with pericarditis.

determined following clinical examination, radiography, and necropsy.

\section{Materials and Methods}

5 cows, diagnosed with chronic pericarditis based on a combination of history, clinical examination, ultrasonography, and radiography, were utilized. Rectal temperature, heart rate, respiration rate, rumen motility, and complete blood cell count were recorded. Plain pericardiocentesis was performed in the left 5 th intercostal space (ICS) at the elbow level by using a sterile hypodermic needle ( $20 \mathrm{G} \times 3.5$ inches). For ultrasonography, the areas extending from the tubercoxae to 3rd ICS and from the dorsal midline to linea alba on both sides were shaved. Animals were secured in standing position in a crate and their various thoracoabdominal organs were examined with a $3.5 \mathrm{MHz}$ curvilinear ultrasonic transducer. Ultrasound-guided pericardial, peritoneal, and pleural paracenteses were performed, and all the aspirated samples were subjected to the microbial culture test. The distances between dorsal midline of the body and dorsal as well as ventral ultrasonographic margins of the liver were measured from 12th to 10th ICS, and compared with the normal liver based on a study conducted by one of the authors (Imran) [12]. Approximated ultrasonographic topography of the liver depicting its dorsoventral and craniocaudal extents was drawn over the right hemiabdomen (Figure 8). Standard radiographs of the thorax and reticulum inlateral recumbency were also obtained. All the cows suffering from the pericarditis died during the course of the treatment and were subjected to necropsy.

\section{Results}

3.1. Clinical Examination. Generally, the history included relapsing fever, inappetance, ventral edema, progressive

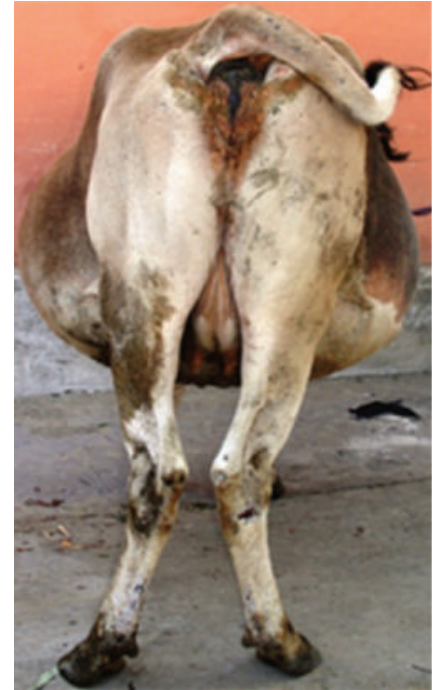

FIgure 2: Classic pear-shaped abdomen, indicative of ascites, in a Jersey cross-bred cow with pericarditis.

abdominal distention, diarrhea, and fall in milk yield ranging from 20 days to 2.5 months. All the animals were refractory to various symptomatic medical treatments attempted at field level. On clinical examination, all the cows were found to be depressed, dyspnoeic, exercise intolerant, and moderately to severely tachycardic.

Bruxism and respiratory grunts on tracheal auscultation were indicative of chronic pain. The muffled heart sounds, bilateral jugular venous distension/pulsation and retrograde refilling, ventral edema (Figure 1), and intermittent diarrhea were recorded in 3 cows and were absent in the rest of the 2 cases. Classic pear-shaped abdomen, indicative of the ascites was observed in 3 of the 5 cows. Paradoxically, the abdominal distention was greatest in one of the cows which did not show the signs of ventral edema and jugular venous distention/pulsation (Figure 2). Ruminal motility was reduced in 3 cows and absent in rest of the 2 cases. Initially, the rectal temperature was within the normal range in 3 cows, and the rest of the 2 cows had fever. However, moderate hypothermia was recorded in the terminal stages of the disease in all the cases. Plain pericardiocentesis revealed foul smelling exudate in 3 of the 5 cows and confirmed the diagnosis of suppurative pericarditis. However, ultrasonographic-guided centesis revealed similar offensive exudate in 4 of the 5 cases. The hematological parameters revealed elevated total leukocytic count, lymphocytopenia, and neutrophilia (Table 1). Microbiological reports of the pericardial aspirates revealed Gram negative bacilli in all the cows. The pleural and peritoneal fluid samples were straw coloured containing fibrin strands, and yet microbiologically sterile.

3.2. Radiographic Observations. Casting of the cows in lateral recumbency for radiography caused severe respiratory distress, which was manifested by open mouth breathing in all the cases. Upon radiographic examination, multiple 
TABLE 1: Clinical parameters recorded in bovine pericarditis.

\begin{tabular}{lccc}
\hline Reference range & Mean clinical values & Reference range & Mean clinical values \\
\hline Rectal temperature $\left(101-102^{\circ} \mathrm{F}\right)$ & 101.1 & Heart rate $(60-80$ beats $/ \mathrm{min})$ & 97.0 \\
Respiration rate $(25$ breaths $/ \mathrm{min})$ & 35.71 & Hemoglobin $(7.5-12.5 \mathrm{~g} / \mathrm{dL})$ & 7.94 \\
Hematocrit $(23-36 \%)$ & 32.43 & Leukocytes $\left(4-20 \times 10^{3} / \mu \mathrm{L}\right)$ & 22.70 \\
Neutrophils $(28 \%)$ & 61.17 & Lymphocytes $(58 \%)$ & 36.83 \\
\hline
\end{tabular}
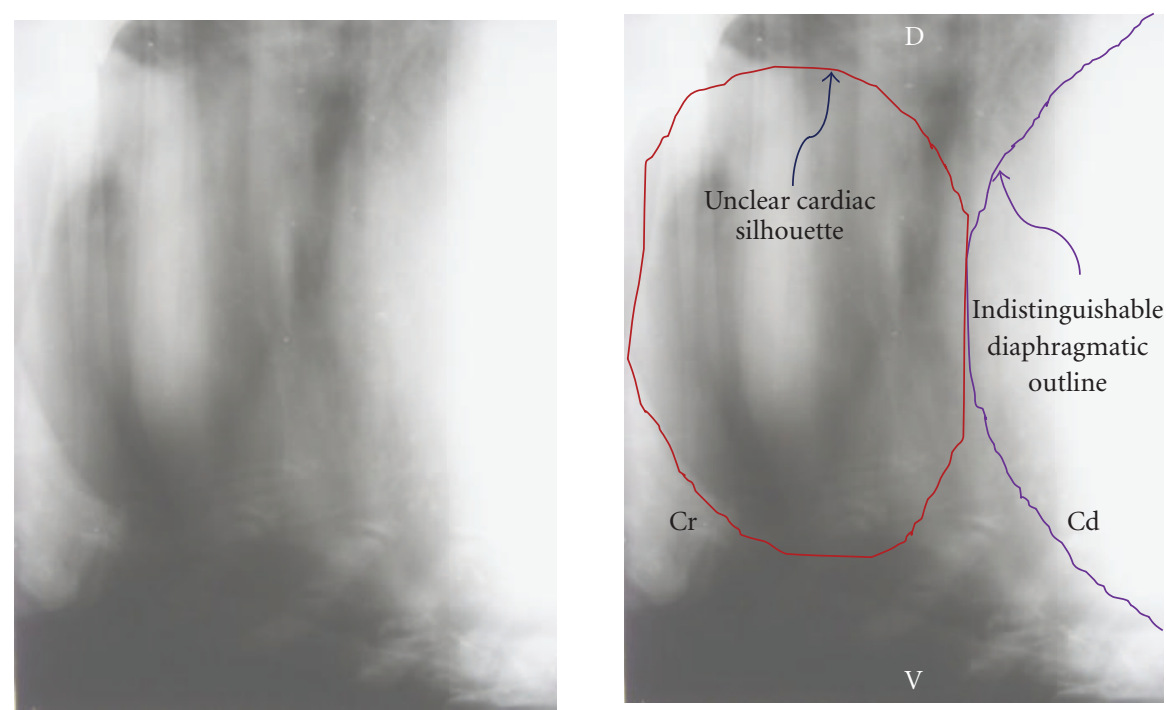

FIGURE 3: Right lateral thoracic radiograph of a pericarditis affected cow depicting indistinguishable cardiac silhouette and diaphragmatic outline. Cr: cranial; Cd: caudal; D: dorsal; V: ventral.

potential metallic foreign bodies were observed inside the area of reticulum and thorax in only 1 cow, whereas no foreign body was detected in the rest of the 4 cases. The main radiographic findings were poor thoracic details, which included indistinguishable diaphragmatic outline and cardiophrenic silhouette (Figure 3).

3.3. Ultrasonographic Observations. When scanned from the right side, the heart was found to be displaced away from the thoracic wall, ranging from 2.8 to $3.6 \mathrm{~cm}$ (mean, $3.4 \mathrm{~cm}$ ) in 4 cases. In these 4 cows, the pericardium was hyperechogenic and markedly thick (mean, $0.8 \mathrm{~cm}$ ). The hyperechogenic pericardium and epicardium were separated by varying quantity of hypoechogenic exudate. Floating echogenic fibrin shreds were often observed with hypoto anechogenic pericardial effusion, and sometimes these shreds bridged epicardium and pericardium. In 2 cases, echogenic sediment was also observed in the ventral most part of the pericardial cavity. A subjective assessment of the reduced size of the cardiac chambers could be appreciated in these 4 cows (Figure 4). The size of the chambers was observed to increase immediately after the gradual removal of the pericardial effusion (Figure 5).

When the heart was ultrasonographically examined from the left side, the evenly spread hypoechogenic pericardial effusion could be seen in only 2 of these 4 cows. Generally, the surface of pericardium was rugged and echogenic pericardial projections were imaged throughout, which were indicative of chronic inflammatory changes (Figure 6). Localized hypoechogenic pericardial cavitary lesions were seen in 2 cows. These were the same 2 cows in which plain pericardiocentesis failed to aspirate any effusion. Anechogenic pleural effusion of varying quantity was also observed in these 4 cows. Free echogenic fibrin shreds were observed occasionally floating within the pleural effusion. Normal internal reverberation pattern of the lungs was observed in the dorsal region of the thorax in all the 5 cows.

In the 5th case, the heart was not visible ultrasonographically from either side. However, its pericardium was imaged as a thick echogenic surface $(1.2 \mathrm{~cm})$ with a distal acoustic shadow, which masked the details of underlying structures and also obscured the optimal site for the drainage of the effusion (Figure 7). Pleural effusion was absent in this case. Thick yellowish pus was aspirated on plain pericardiocentesis, which confirmed the diagnosis.

Markedly enlarged liver, extending from just below the dorsal midline to linea alba and cranially up to the 5th ICS, was a noticeable ultrasonographic feature in all the cows (Figure 8, Table 2). Slight increase in echogenicity of the ventral part of liver was also noticed. The gallbladder 

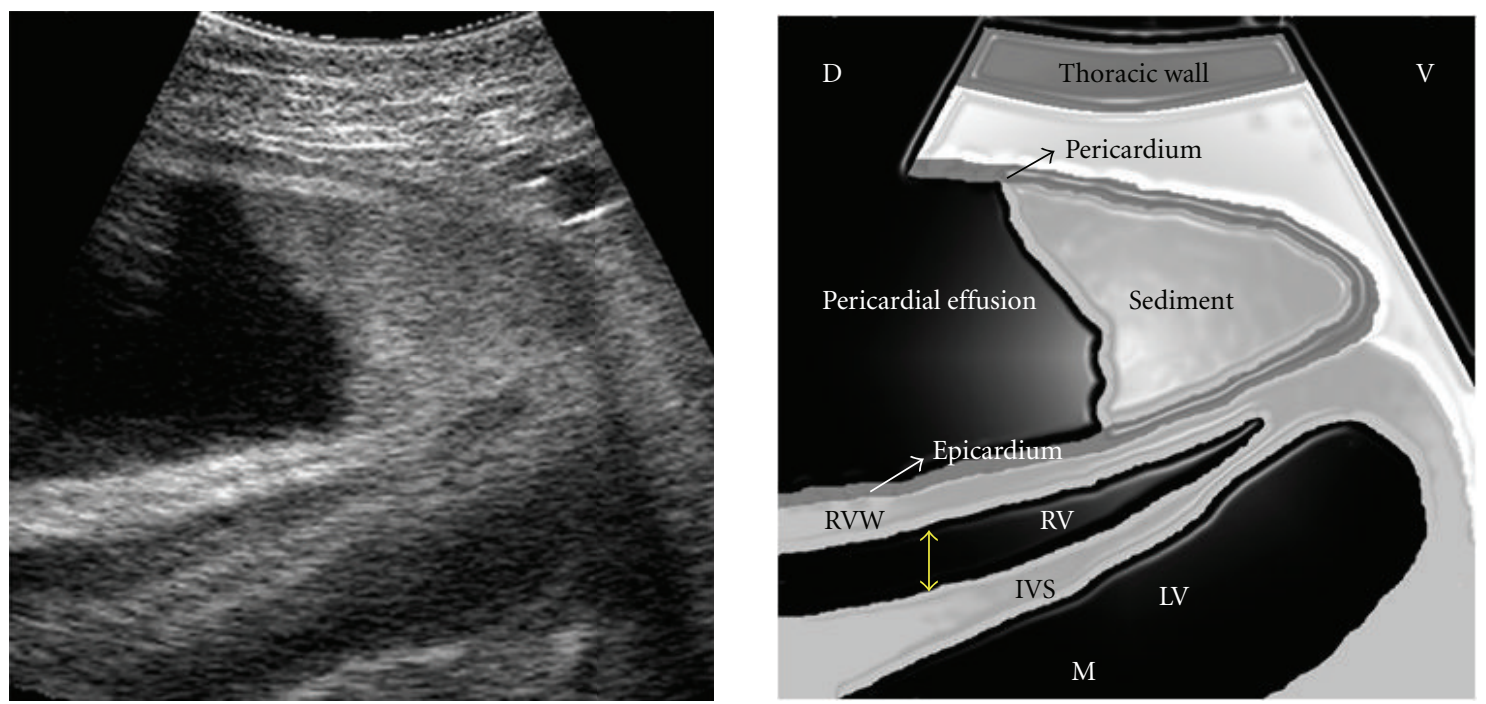

FIgURE 4: Two-dimensional echocardiogram obtained from right 4th ICS, by placing the transducer parallel to the ribs, depicting anechogenic pericardial effusion and echogenic sediment at the apex of pericardial cavity. Notice the compression of the right ventricle. ICS: intercostal space; RVW: right ventricular wall; IVS: interventricular septum; RV: right ventricle; LV: left ventricle; D: dorsal; V: ventral; M: medial.
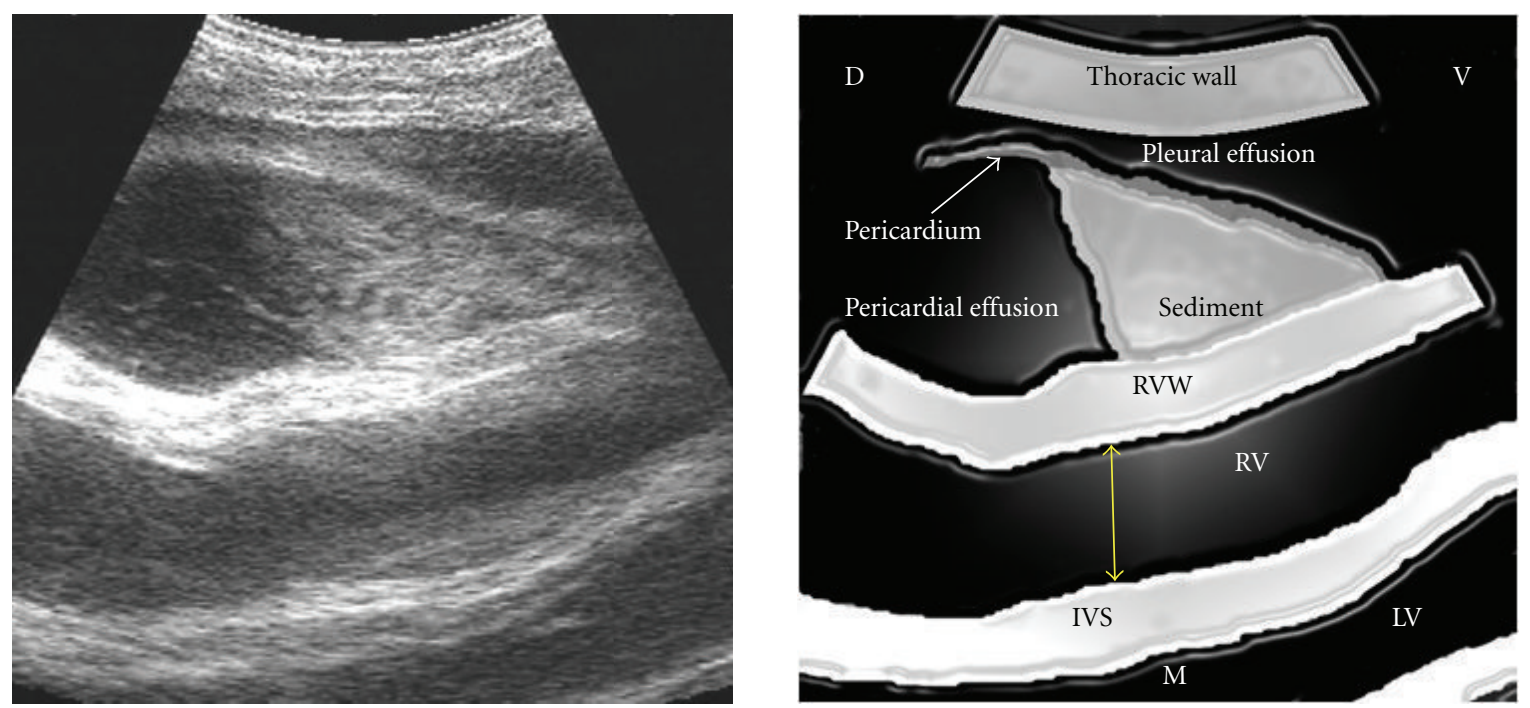

FIGURE 5: Two-dimensional echocardiogram obtained after ultrasound-guided aspiration of the pericardial effusion from the right 4th ICS, by placing the transducer parallel to the ribs. Notice the increase in the size of the right ventricle.

was slightly distended and its wall was thick and typically double layered, indicative of chronic edema (Figure 9). The distension of the caudal vena cava (CVC) was severe and its diameter ranged from 3.8 to $4.3 \mathrm{~cm}$ (mean, $4.12 \mathrm{~cm}$ ), when viewed from the 12th or 11th ICS (Figure 10). The shape of the CVC was oval to circular in cross-section. The hepatic veins in the vicinity of the CVC were also dilated. The portal vein was normal in 3 cows and poorly visible in 2 cows.

Other noticeable changes were accumulation of the varying degree of anechogenic fluid in the peritoneal cavity in all the cases, indicative of ascites. The parietal layer of the peritoneum was seen just adjacent to the body wall as an echogenic line, undulating with each respiratory movement.

The abdominal visceral organs were seen submerged or floating in the peritoneal fluid (Figures 11 and 12). Echogenic fibrinous deposits on the surface of reticulum, rumen, liver, and spleen were also observed (Figures 13 and 14). Reduced amplitude and frequency (approximately 1 per 2 minutes) of first reticular contraction was observed in 3 cows, and atonic reticulum was seen in the rest of the 2 cows. Foreign 

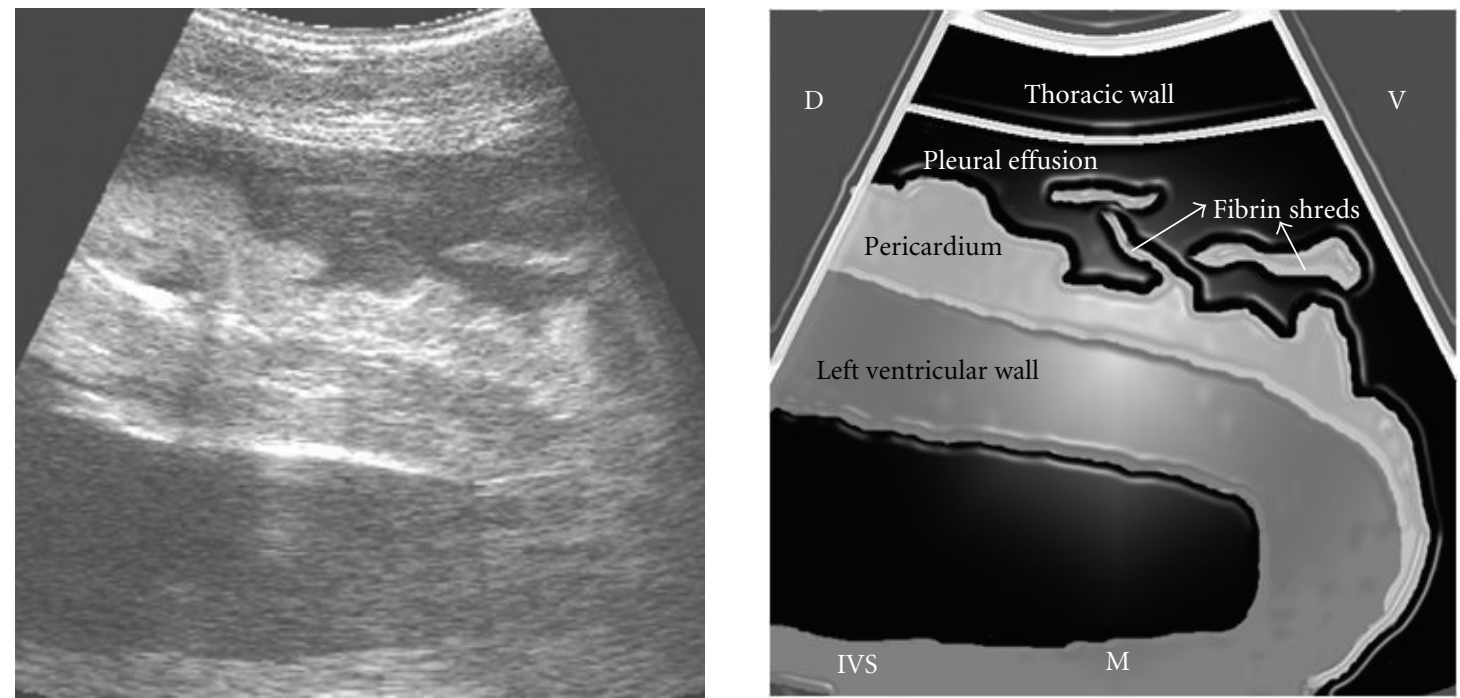

FIGURE 6: Two-dimensional echocardiogram obtained from the left 5th ICS, showing pleural effusion and thickened pericardium with fibrin shreds deposited on its surface. ICS: intercostal space; IVS: interventricular septum; D: dorsal; V: ventral; M: medial.
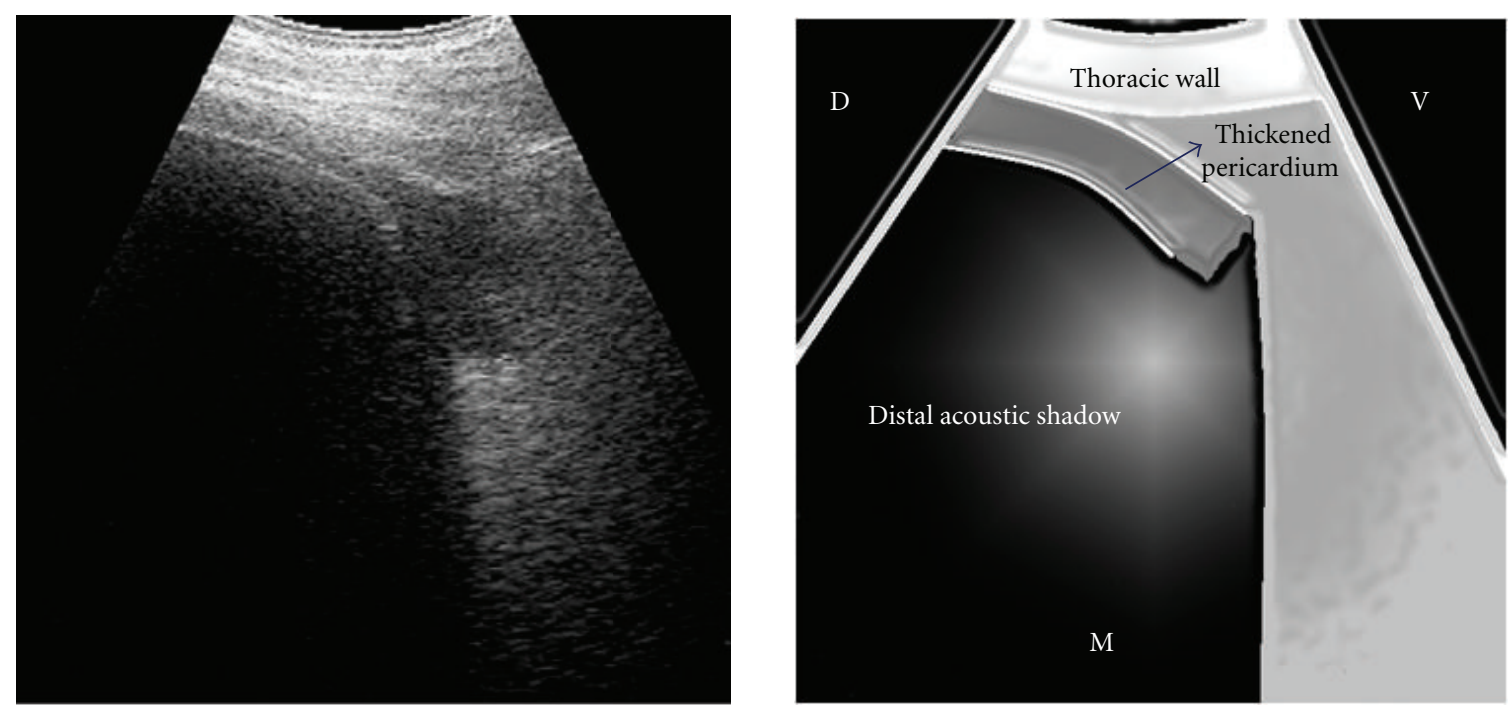

FIgURE 7: Two-dimensional echocardiogram obtained from the right 4th ICS, showing thickened pericardium with distal acoustic shadow. ICS: intercostal space; D: dorsal; V: ventral; M: medial.

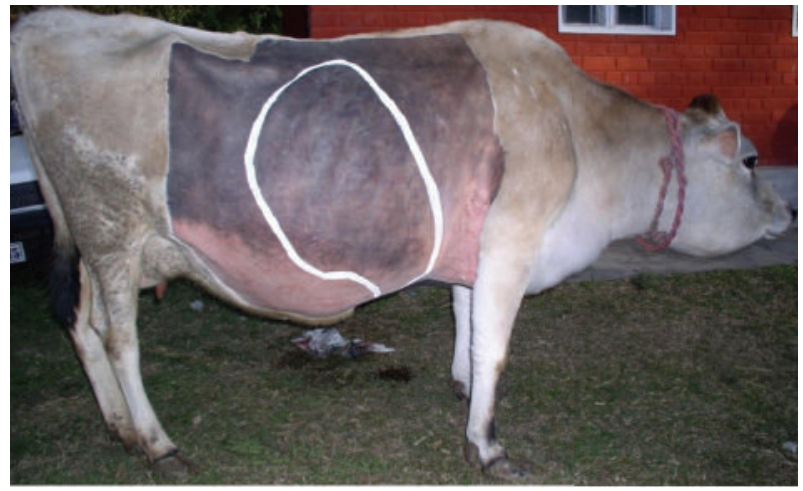

FIGURE 8: Ultrasonographic topography of the enlarged liver based on its mean dorsoventral extent in 5 cows affected by pericarditis.
TABLE 2: Ultrasonographically determined mean dorsoventral extent of the liver in cows.

\begin{tabular}{lccc}
\hline & \multicolumn{3}{c}{ Dorsoventral extent $(\mathrm{cm})$} \\
& 12th ICS & 11th ICS & 10th ICS \\
\hline Cows with pericarditis $(n=5)$ & 50.33 & 51.33 & 45.34 \\
Normal cows [12] $(n=10)$ & 24.2 & 25.6 & 17.3 \\
\hline
\end{tabular}

bodies seen radiographically in 1 cow could not be imaged ultrasonographically. The ventral body wall was seen as consisting of alternating echogenic to hypoechogenic areas, resembling a reticulate pattern, consistent with muscles and the edematous fluid. 

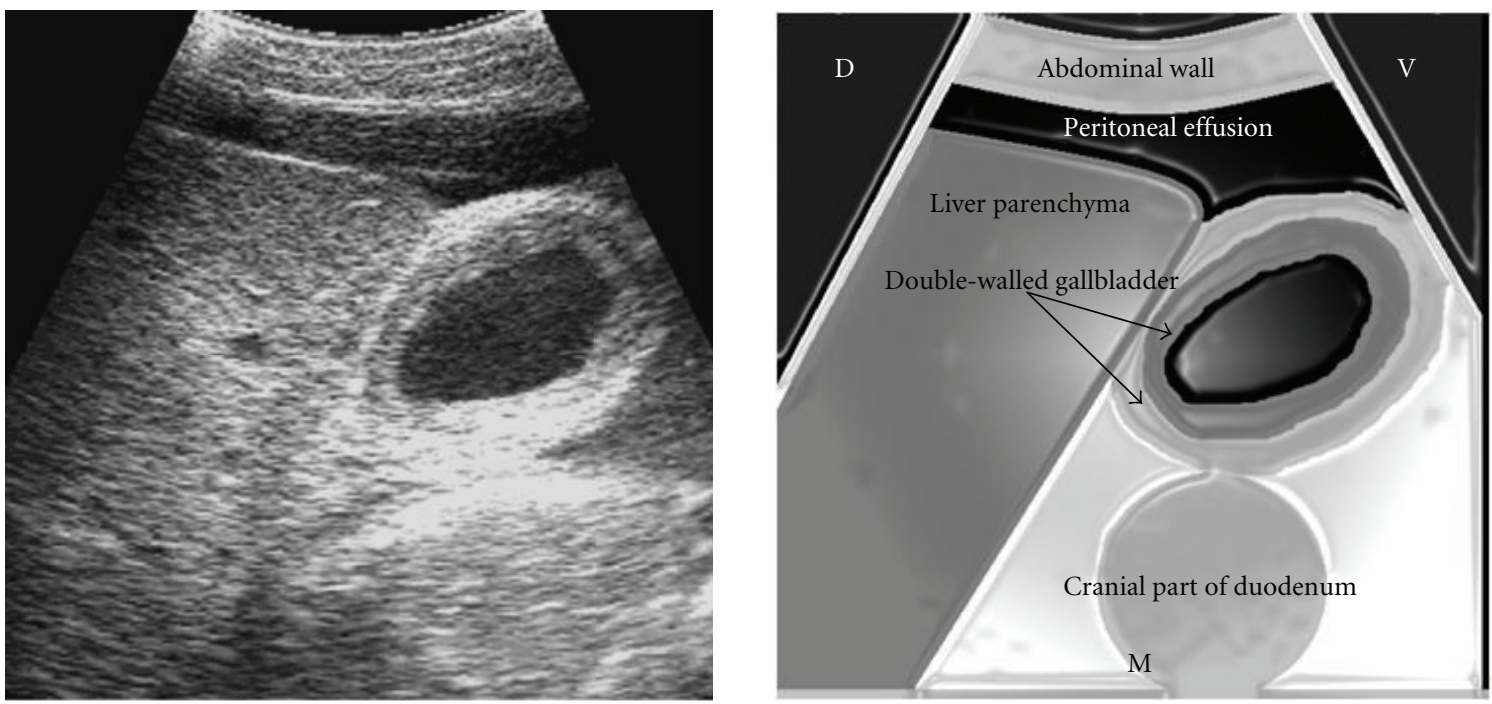

FIGURE 9: Ultrasonogram showing the edematous double-walled gallbladder, obtained from the 10th ICS. CVC: caudal vena cava; ICS: intercostal space; D: dorsal; V: ventral; M: medial.
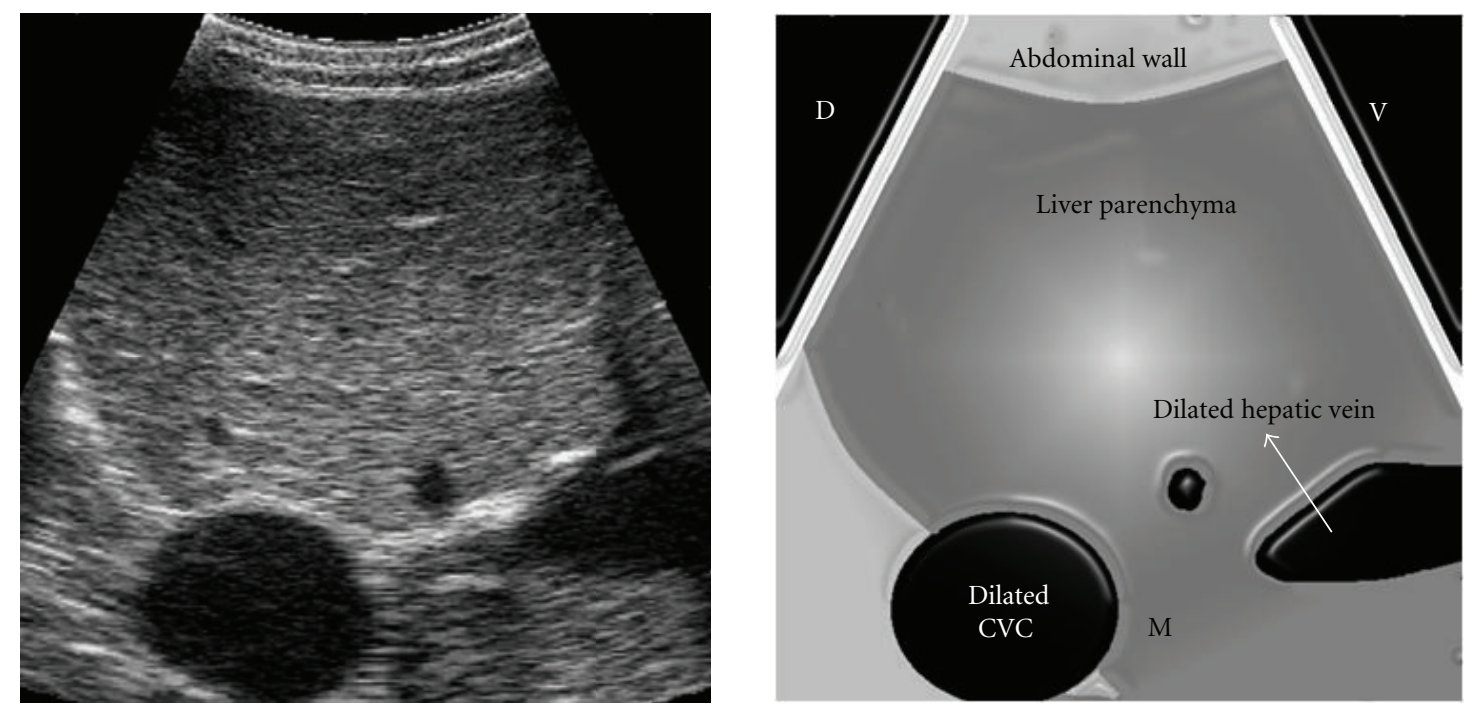

FIGURE 10: Ultrasonogram showing the congested CVC, obtained from the 12th ICS. CVC: caudal vena cava; ICS: intercostal space; D: dorsal; $\mathrm{V}$ : ventral; M: medial.

3.4. Necropsy. Necropsy confirmed the diagnosis of chronic suppurative pericarditis in all cases (Figure 15). Foreign body could be retrieved from pericardium and reticulum in only 1 cow. This was the same cow in which foreign bodies were detected by radiography. Pleural effusion was observed in only those 4 cows, which were previously diagnosed by ultrasonography. Peritoneal effusion, hepatomegaly, and congestion of the liver were also observed in all the cases (Figures 16, 17, and 18).

\section{Discussion}

In our study, a tentative diagnosis of the pericarditis was made on the basis of their characteristic clinical signs which included a mean heart rate of $97 \mathrm{bpm}$, distension/pulsation of jugular veins and muffled heart sounds. The clinical signs shown by the animals differed because of variations in the severity of the disease. As the volume of fluid within the pericardial sac increased, so did the severity of cardiac compression. This lead to a vicious cycle of reduced venous return induced increase in the venous hydrostatic pressure, ultimately resulting in progressive peritoneal effusion, clinically manifested as ascites.

Hematological examination demonstrated significant leukocytosis with neutrophilia and lymphocytopenia. A marked leukocytosis and neutrophilia are usually present in traumatic pericarditis, because this has many of the characteristics of a large internal abscess [13]. 

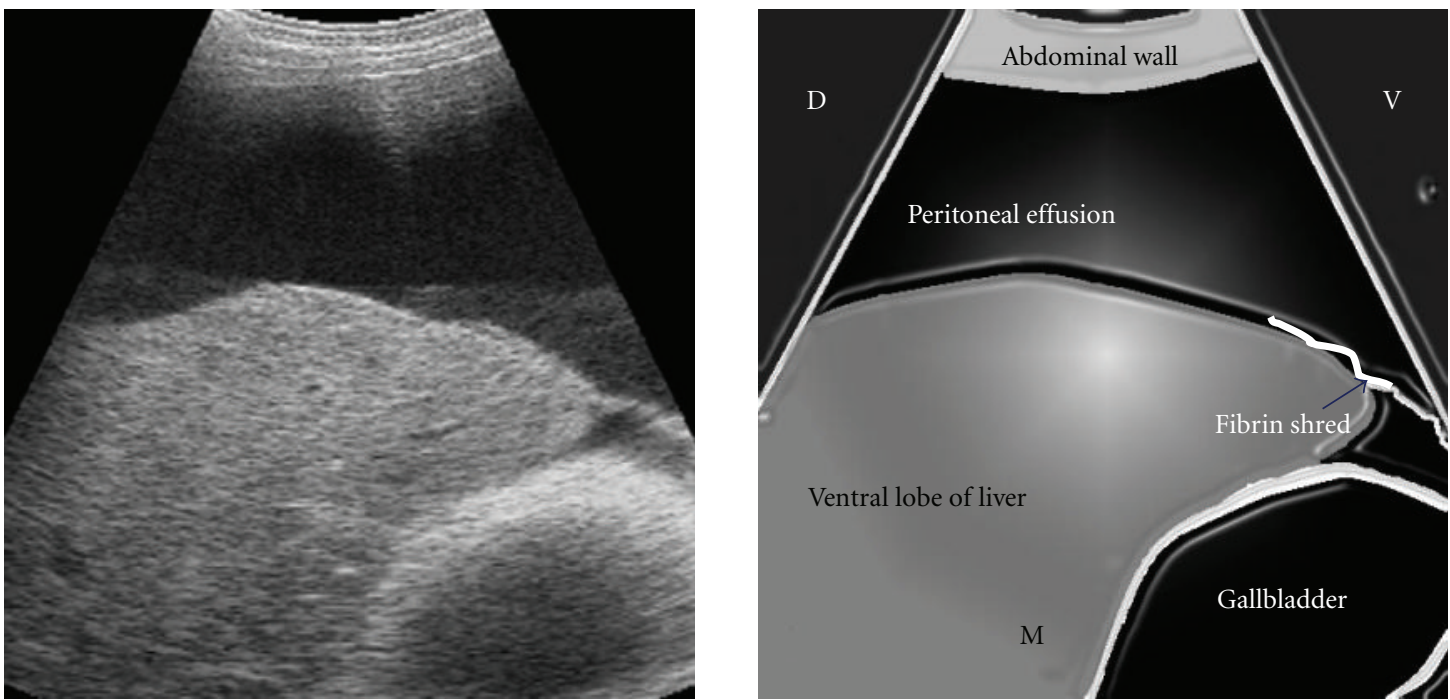

FIGURE 11: Ultrasonogram of the liver submerged in peritoneal effusion, with fibrin shred on its parietal surface, viewed from the right 11 th ICS. ICS: intercostal space; D: dorsal; V: ventral; M: medial.
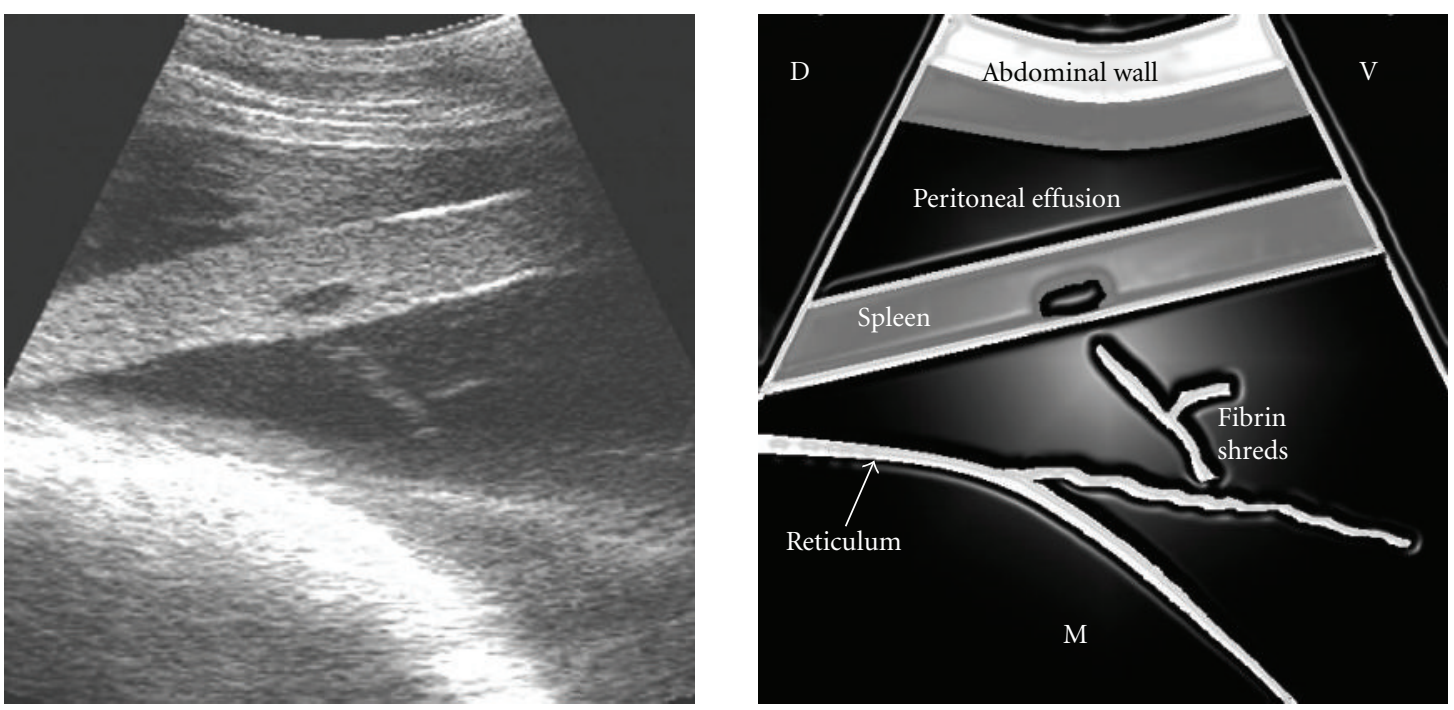

FIGURE 12: Ultrasonogram of the reticulum and spleen showing peritoneal effusion along with fibrin shreds viewed from the left 7th ICS. Notice the peritoneal effusion induced acoustic enhancement, depicted by the hyperechogenicity of the reticular wall. ICS: intercostal space; D: dorsal; V: ventral; M: medial.

Traumatic pericarditis in cattle is most commonly caused by penetrating metallic foreign bodies either migrating from reticulum or due to impaling [14]; yet, the foreign bodies are not seen radiographically in as much as $76 \%$ of pericarditis cases [15]. This was reported due to masking of their image by severe opacity created by suppurative pericarditis. However, in the present study, the foreign bodies were detected radiographically in only 1 cow, and these findings coincided with the postmortem examination. This indicated that true absence of foreign bodies was the reason for negative radiographic diagnosis in the other 4 cases. Occasionally, a diagnosis of traumatic pericarditis is based on characteristic lesions alone when no foreign body can be found on the radiographs or at necropsy [10].

Plain pericardiocentesis may fail to aspirate accumulated pericardial effusion, especially if it is done only from one side. It happened in 2 cases of the present study, where plain pericardiocentesis was done from the left side alone and revealed nothing. Ultrasound-guided pericardiocentesis safely drained septic effusion in these 2 cows as well. This implies that plain pericardiocentesis should be done from both the sides of thorax to rule out the pericardial effusion effectively. Further, complications associated with plain 

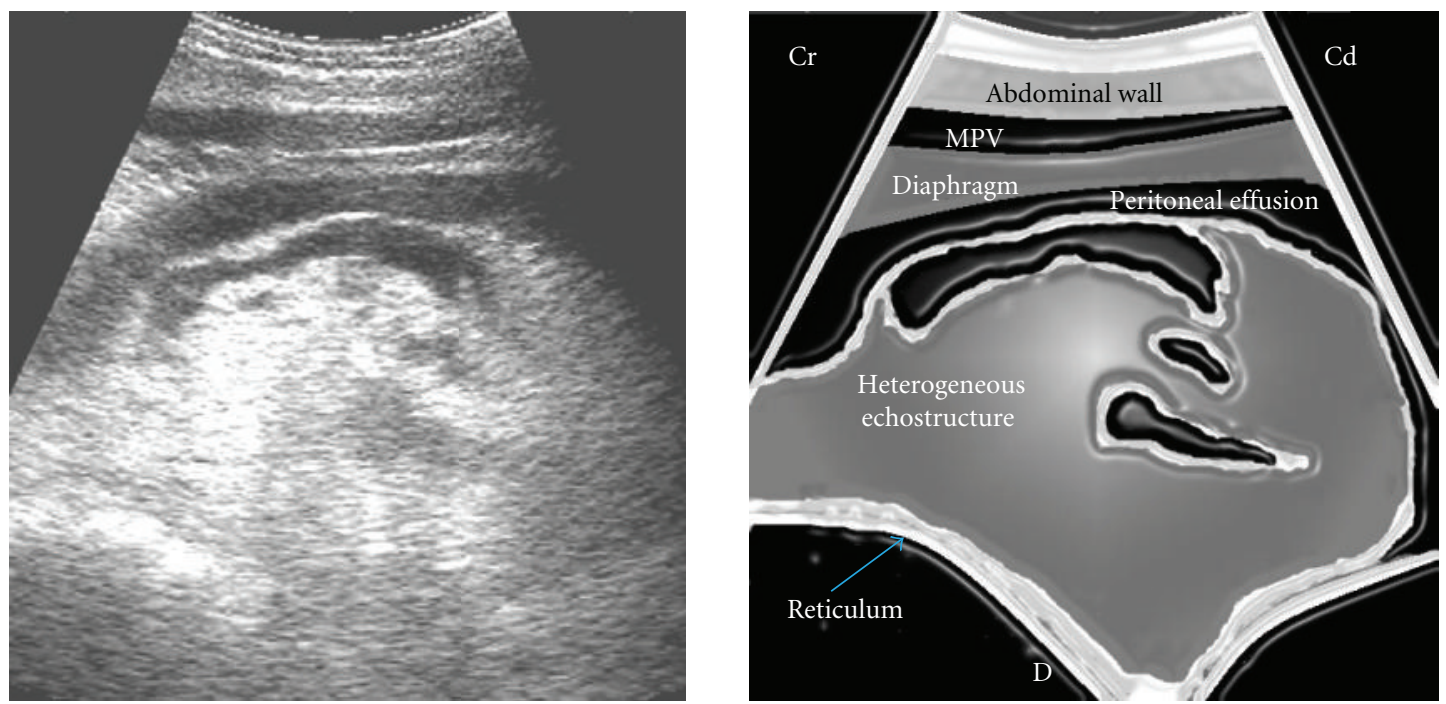

FIGURE 13: Left paramedian sonogram depicting deposition of the echogenic fibrin interspersed with the hypoechogenic fluid between the diaphragm and reticulum. MPV: musculophrenic vein; Cr: cranial; Cd: caudal; D: dorsal.
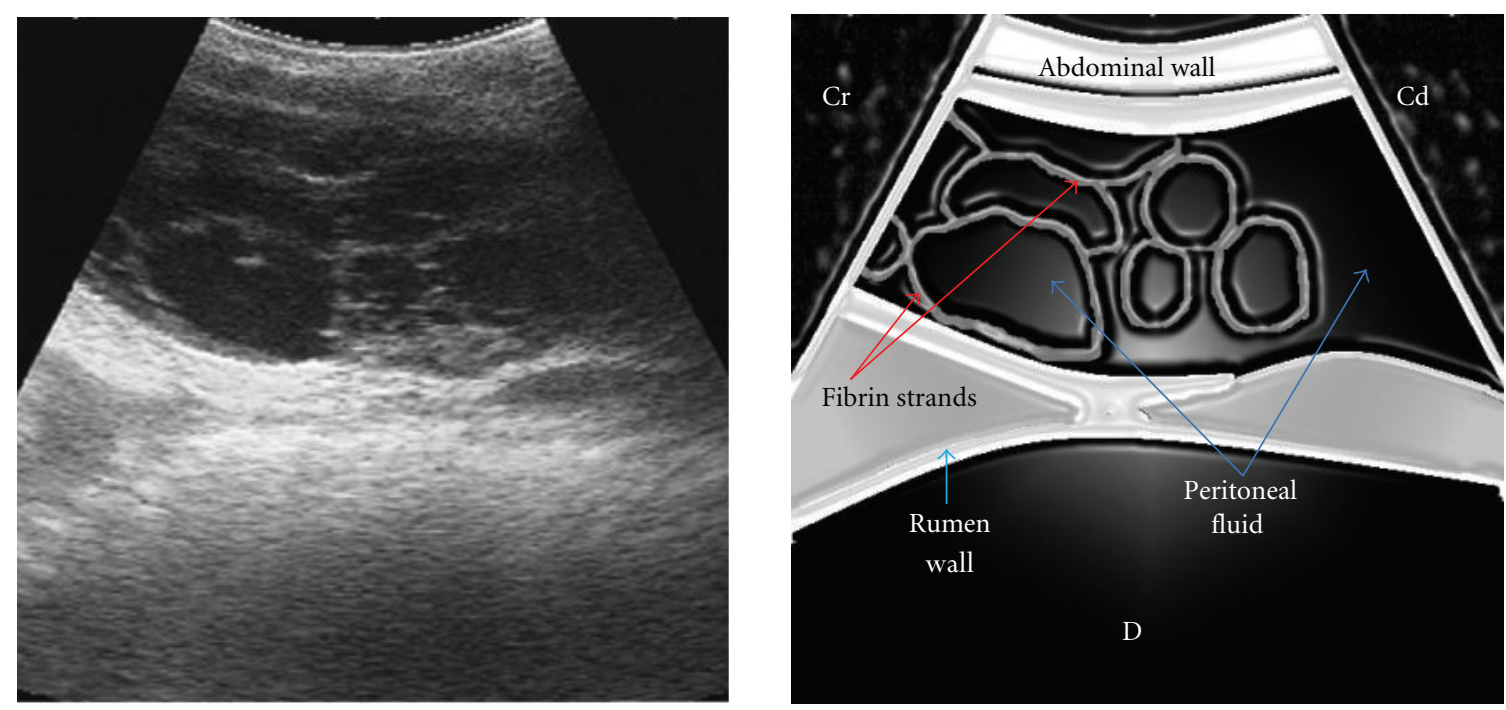

FIGURE 14: Left paramedian sonogram depicting the deposition of the echogenic fibrin along with hypoechogenic fluid between the ventral abdominal wall and rumen. Cr: cranial; Cd: caudal; D: dorsal.

pericarditis can be mitigated by performing the procedure using ultrasonographic guidance [5].

There are some limitations of the ultrasonography as well. For example, in 1 of the 5 cows, details of the structures beyond the pericardium could not be seen due to distal acoustic shadow. Although plain pericardiocentesis revealed foul smelling exudate, the pericardial sac was not visible ultrasonographically. The distal acoustic shadow is known to occur due to greater difference in acoustic impedance at the gas-tissue interface [16]. Gas produced by the bacteria in the pericardium acts to inflate the pericardium away from the heart, thereby lessening possible restrictive pressures [5]. The gas produced by bacteria in the pericardium was considered to be responsible for the accumulation of gas in this particular case, as also indicated by thoracic radiography and microbial culture.

The compression of the cardiac chambers was also a prominent feature in cases of the pericarditis, which has been reported [10]. The decompression of the chambers following the partial removal of pericardial effusion did not help in alleviating the cows' pathetic condition, since all the cows collapsed within 5-8 days of the referral. Cardiac tamponade is a life-threatening hemodynamic condition resulting from pericardial effusions that increase intrapericardial pressure sufficiently to externally compress and restrict cardiac chamber filling, constrain cardiac output, and induce backward 


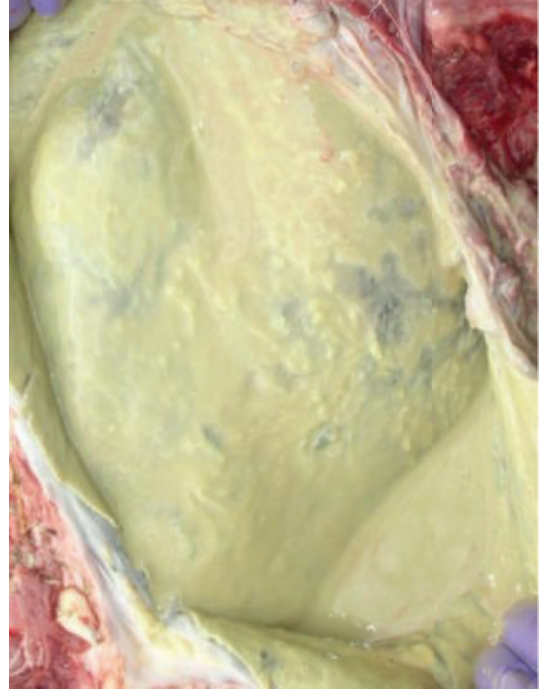

FIGURE 15: Thick pericardium along with septic effusion.

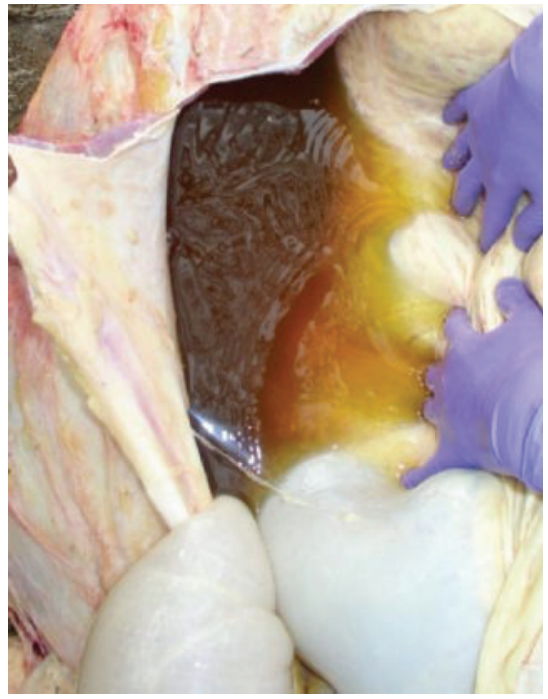

Figure 16: Postmortem view of the ascites.

failure. Hemodynamically significant effusions compress the cardiac chambers, particularly the thinner more compliant right heart chambers, throughout diastole [17]. Mean right ventricular diastolic and intrapericardial fluid pressures are increased in a corresponding manner in cows with clinical signs of the right-sided heart failure [9]. Cattle with muffled heart sounds and a large pericardial fluid volume also have a decrease in cardiac output to approximately two-thirds of normal values [9]. Most animals with the signs of congestive heart failure die within 1 or 2 weeks [6].

Reduced right ventricular diastolic filling increases the venous hydrostatic pressure, which leads to the hepatic enlargement, congestion, as well as distension of the jugular veins and the CVC. This is the logical explanation why the CVC assumed oval to round shape in the cows with heart failure instead of being triangular shaped, as seen in

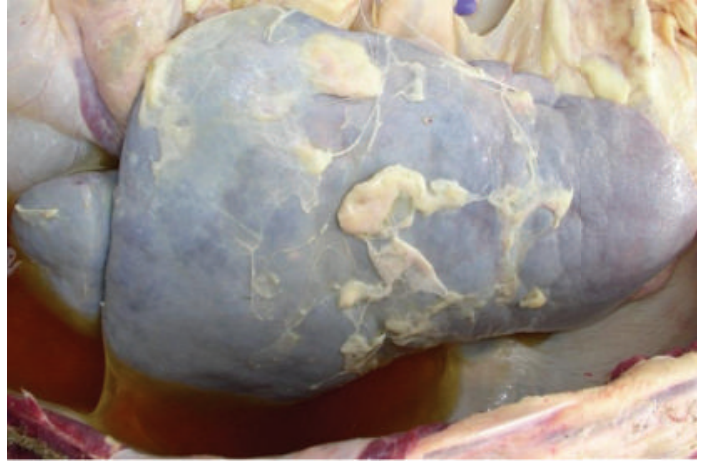

Figure 17: Postmortem view of the hepatomegaly along with fibrin shreds on its parietal surface.

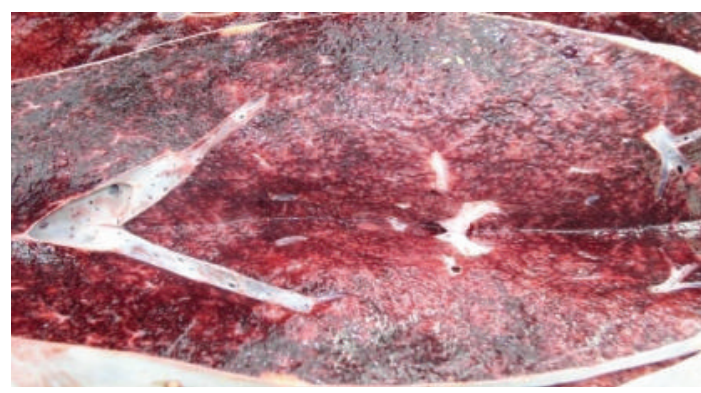

FIGURE 18: Postmortem appearance of the congested liver, indicative of chronic heart failure.

the healthy cows [12]. Acoustic enhancement due to the ascitic fluid was the reason for the increased echogenicity of the ventral part of the liver. Distal acoustic enhancement is caused by augmentation of the amplitude of the echoes distally to a structure with a low attenuation, more often fluid [16]. All these features are associated with the congestive heart failure usually seen in terminal stages of the pericarditis [10]. Therefore, ultrasonography can be a useful prognostic tool in such cases. Moreover, intermittent diarrhea observed in 3 cows can also be attributed to the congestive heart failure, since hepatic venous congestion is known to impair the digestion and absorption [9].

Based on the poor prognosis, under normal circumstances, cattle with traumatic pericarditis should be slaughtered or euthanased rather than treated $[10,13]$. Treatment should be attempted only in a valuable animal or in an animal carrying a high value embryo [10]. However, many farmers in India prefer palliative treatment of the cows with pericarditis, irrespective of the cost-benefit analysis, owing to sanctity of the cow in Hinduism. In our opinion, the clinician should be aware of the dialectics between veterinary ethics and traditionalism before making a final decision.

In conclusion, the clinical and radiographic findings did not allow a definitive diagnosis of the pericarditis, since all of the characteristic signs were not present in every case. Thoracoabdominal ultrasonography played an important role in the early diagnosis and prognosis of the pericarditis, owing to its direct and noninvasive analysis of the pericardial effusion and the hepatic congestion. 


\section{References}

[1] T. C. Jones, R. D. Hunt, and N. W. King, "Cardiovascular system," in Veterinary Pathology, Lippincott Williams \& Wilkins, Philadelphia, Pa, USA, 3rd edition, 1996.

[2] V. B. Reef and S. M. McGuirk, "Diseases of the cardiovascular system," in Large Animal Internal Medicine, B. P. Smith, Ed., pp. 95-97, 463-466, Mosby, St. Louis, Mo, USA, 3rd edition, 2002.

[3] S. Buczinski, A. Rezakhani, and D. Boerboom, "Heart disease in cattle: diagnosis, therapeutic approaches and prognosis," Veterinary Journal, vol. 184, no. 3, pp. 258-263, 2010.

[4] D. Krishnamurthy, "Hernia," in Ruminant Surgery, R. P. S. Tyagi and J. Singh, Eds., pp. 256-259, CBS Publishers, New Delhi, India, 1st edition, 1993.

[5] S. F. Peek and S. M. McGuirk, "Examination and assessment, Cardiovascular diseases," in Rebhun's Diseases of Dairy Cattle, pp. 30,53-55, WB Saunders, Philadelphia, Pa, USA, 2nd edition, 2008.

[6] O. Ramakrishna, "Cardiovascular system," in Ruminant Surgery, R. P. S. Tyagi and J. Singh, Eds., pp. 256-259, CBS Publishers, New Delhi, India, 1st edition, 1993.

[7] M. B. Gavali, V. D. Aher, and A. U. Bhikane, "Surgical management of traumatic pericarditis in bovine-a clinical study," Indian Veterinary Journal, vol. 80, no. 6, pp. 556-559, 2003.

[8] S. A. Jesty, R. W. Sweeney, B. A. Dolente, and V. B. Reef, "Idiopathic pericarditis and cardiac tamponade in two cows," Journal of the American Veterinary Medical Association, vol. 226, no. 9, pp. 1555-1558, 2005.

[9] O. M. Radostits, C. C. Gay, K. W. Hinchclif, and P. D. Constable, "Manifestations of circulatory failure," in Veterinary Medicine: A Textbook of the Diseases of Cattle, Horses, Sheep, Pigs and Goats, pp. 402-431, Elsevier Health Sciences, Philadelphia, PA, USA, 10th edition, 2008.

[10] U. Braun, "Traumatic pericarditis in cattle: clinical, radiographic and ultrasonographic findings," Veterinary Journal, vol. 182, no. 2, pp. 176-186, 2009.

[11] U. Braun, B. Lejeune, S. Rauch, U. Gorber, and G. Schweizer, "Ultrasonographic findings in 22 cattle with pericarditis traumaticaSonographische befunde bei 22 rindern mit pericarditis traumatica," Schweizer Archiv fur Tierheilkunde, vol. 150, no. 6, pp. 281-286, 2008.

[12] S. Imran, Ultrasonography of bovine abdominal cavity, MVSc thesis, CSK Himachal Pradesh Krishi Vishvavidyalaya, India, 2010.

[13] U. Braun, B. Lejeune, G. Schweizer, M. Puorger, and F. Ehrensperger, "Clinical findings in 28 cattle with traumatic pericarditis," Veterinary Record, vol. 161, no. 16, pp. 558-563, 2007.

[14] J. E. Sojka, M. R. White, W. R. Widmer, and W. G. VanAlstine, "An unusual case of traumatic pericarditis in a cow," Journal of Veterinary Diagnostic Investigation, vol. 2, no. 2, pp. 139-142, 1990.

[15] N. A. Misk and M. A. Semieka, "The radiographic appearance of reticular diaphragmatic herniation and traumatic pericarditis in buffaloes and cattle," Veterinary Radiology and Ultrasound, vol. 42, no. 5, pp. 426-430, 2001.

[16] L. Blond and S. Buczinski, "Basis of ultrasound imaging and the mainartifactsin bovine medicine," Veterinary Clinics of North America, Food Animal Practice, vol. 25, pp. 553-565, 2009.
[17] J. A. Goldstein, "Cardiac tamponade, constrictive pericarditis, and restrictive cardiomyopathy," Current Problems in Cardiology, vol. 29, no. 9, pp. 503-567, 2004. 

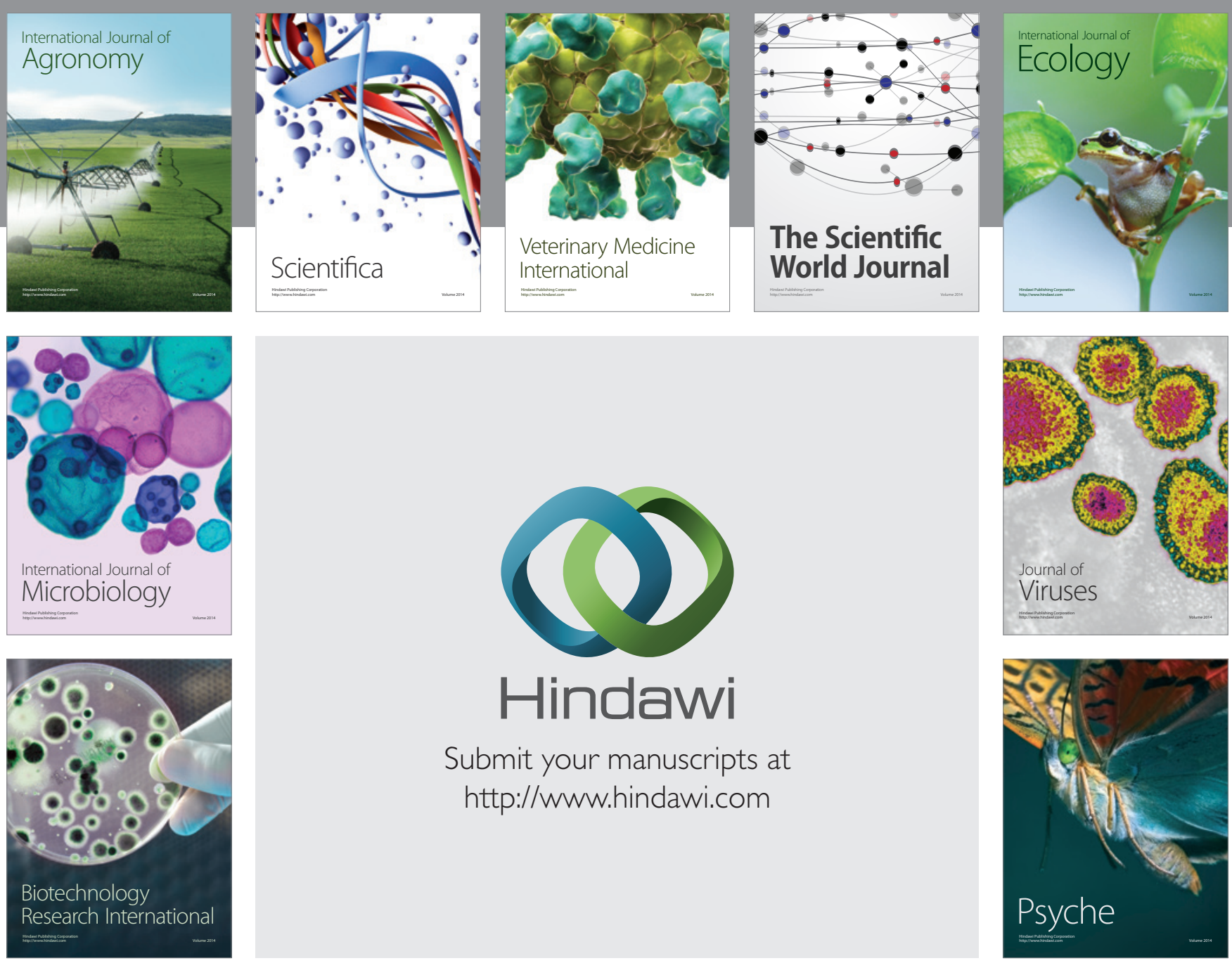

Submit your manuscripts at

http://www.hindawi.com
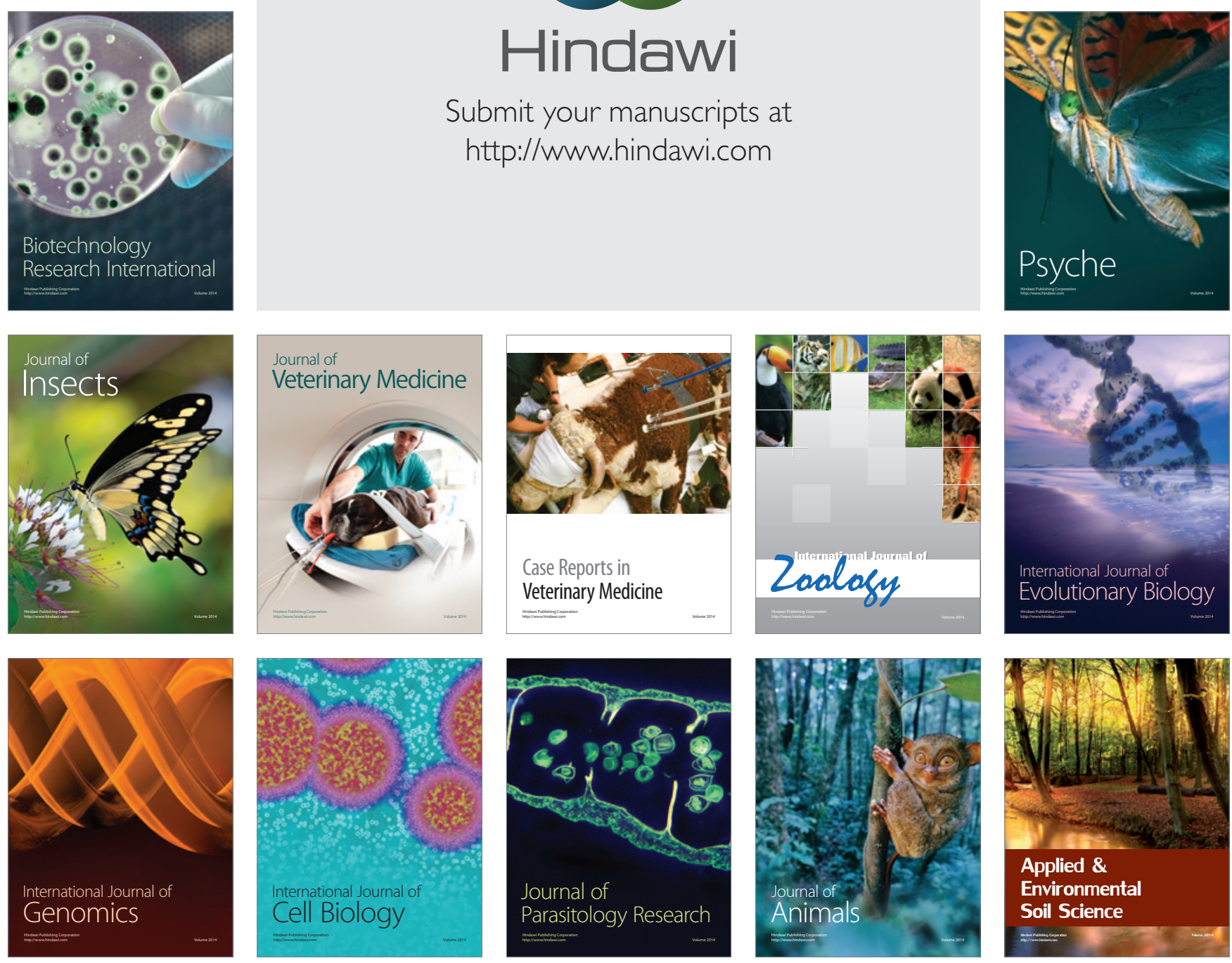